\title{
Radial single-layer nanotubes
}

SIR - Several novel forms of carbon have been discovered since the mid-1980s, including fullerenes ${ }^{1}$, nanometre-sized nested graphitic tubes and polyhedra ${ }^{2}$, and single-layer carbon nanotubes ${ }^{3,4}$. These single-layer tubes have so far been observed only in the primary soots from arc-discharge experiments where the anodes were packed with the ferromagnetic elements Fe (ref. 3), Co (ref. 4) and $\mathrm{Ni}$ (which was observed by our group). Here we report the discovery of clusters of single-layer tubes arranged around nuclei of $\mathrm{Gd}_{x} \mathrm{C}_{y}$ in patterns similar to sea-urchin spines. They are present in large quantities in the primary soots from arcdischarge experiments using either $\mathrm{Gd}_{2} \mathrm{O}_{3}$

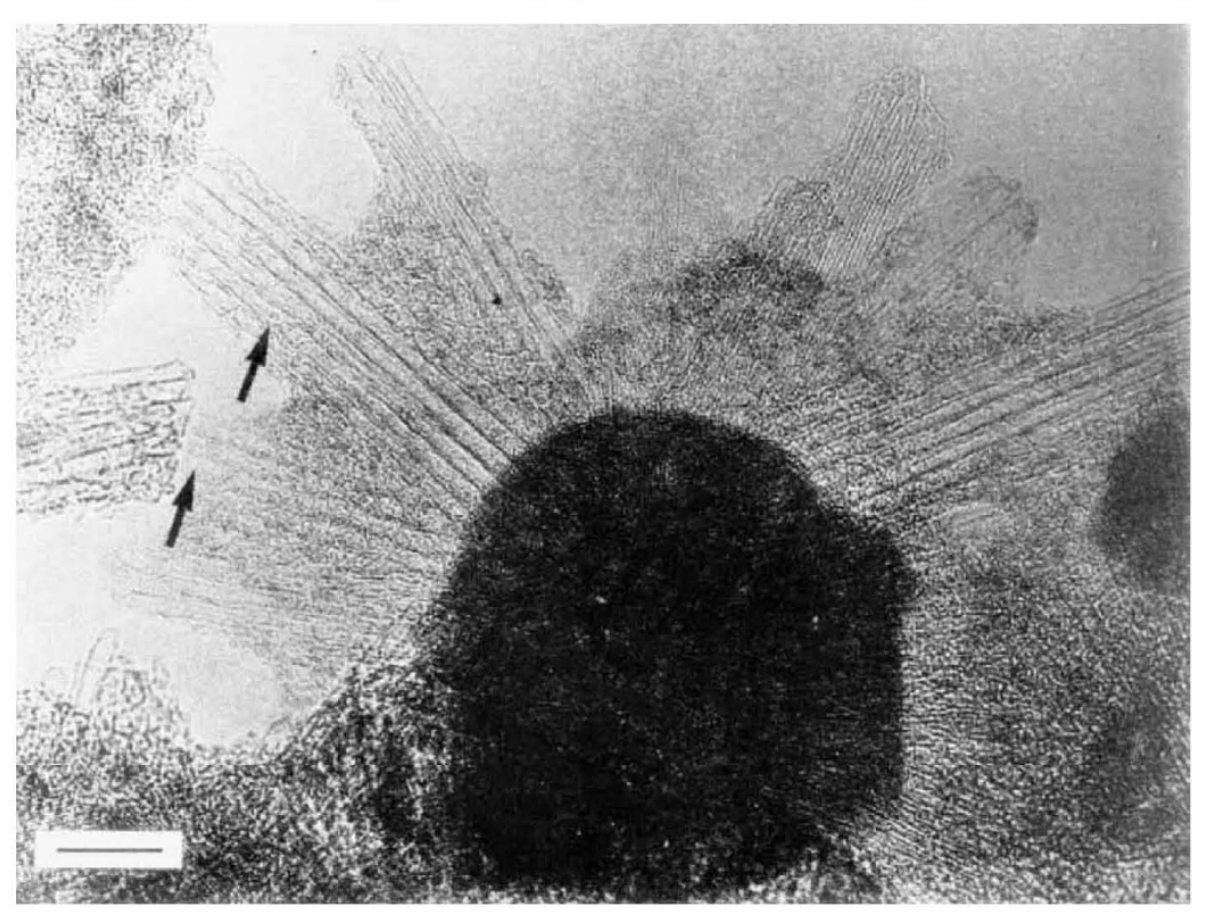

High-resolution transmission electron microscope image of bundles of short predominantly single-layer carbon tubes encapsulating an amorphous core of $\mathrm{Gd}_{x} \mathrm{C}_{y}$. Scale bar, $10 \mathrm{~nm}$. Arrows, possible multi-wall tubes. The microscope is a Phillips CM20 ultra-high resolution TEM.

or pure metal Gd in the anode.

The soot samples described here were prepared in a manner similar to that described earlier ${ }^{5}$. The graphite anode was drilled out and packed with either $\mathrm{Gd}_{2} \mathrm{O}_{3}$ or $\mathrm{Gd}$ such that the $\mathrm{Gd} / \mathrm{C}$ ratio was between 3 and 6 atom $\%$. The cathode is a 12.7-mm diameter graphite rod, and the anode a 8 -mm diameter graphite rod with a $3.2-\mathrm{mm}$ bore drilled out in the centre. The arc gap was typically $3-6 \mathrm{~mm}$, and the current and pressure were $85 \mathrm{amp}$ and 500 torr $\left(\mathrm{Gd}_{2} \mathrm{O}_{3}\right)$ or $75 \mathrm{amp}$ and 1,000 torr (pure $\mathrm{Gd}$ ). The primary soot obtained was dispersed ultrasonically in ethanol and placed on holey carbon-coated $\mathrm{Cu}$ grids for transmission electron microscopy.

The soot morphology can be broadly classified into two types - pure amorphous carbon particles; and amorphous

carbon regions containing substantial quantities of high electron contrast metal nuclei. Energy dispersive spectra confirm that these nuclei are Gd compounds. Careful observation of the regions containing the $\mathrm{Gd}_{x} \mathrm{C}_{y}$ particles shows the prevalence of a 'sea-urchin' type morphology, with the $\mathrm{Gd}_{x} \mathrm{C}_{y}$ particles forming the nuclei of these radial structures.

The figure shows a high resolution image of one of the 'sea urchin' particles, clearly illustrating the bundles of rather short $(<75 \mathrm{~nm})$ single-layer tubes radiating out from a $\mathrm{Gd}_{x} \mathrm{C}_{y}$ core, which appears to be amorphous. Some of the bundles also appear to contain what look like multi-layered tubes, as indicated by

molti-laycred tubes, as 